\title{
Strain-rate-dependent model for the dynamic compression of elastoplastic spheres
}

\author{
Hayden A. Burgoyne ${ }^{1}$ and Chiara Daraio ${ }^{1,2}$ \\ ${ }^{1}$ Graduate Aerospace Laboratories (GALCIT), California Institute of Technology, Pasadena, California 91125, USA \\ ${ }^{2}$ Chair in Mechanics and Materials, ETH Zurich, Zurich 8092, Switzerland
}

(Received 3 January 2014; published 12 March 2014)

\begin{abstract}
We present a force-displacement contact model for the compressive loading of elastoplastic spheres. This model builds from the well known Hertz contact law for elastic, quasistatic compression to incorporate a material's strain-rate-dependent plasticity in order to describe collisions between particles. In the quasistatic regime, finite-element analysis is used to derive an empirical function of the material properties. A Johnson-Cook strain rate dependence is then included into the model to study dynamic effects. We validate the model using split Hopkinson bar experiments and show that the model can accurately simulate the force-displacement response of strain-rate-dependent elastoplastic spheres during dynamic compression and unloading.
\end{abstract}

DOI: 10.1103/PhysRevE.89.032203

PACS number(s): 45.70.-n, 46.40.Cd, 43.25.+y

\section{INTRODUCTION}

Understanding the contact law between spherical bodies has been a fundamental problem in mechanics since Hertz first solved analytically the force-displacement relation for elastic objects [1]. While this interaction is well understood for elastic bodies, the elastoplastic contact deformation of spheres is still not well characterized. The contact mechanics of elastoplastic spheres is relevant in several physical phenomena and engineering applications, for example planetary ring formation, nanoparticle mechanics, high performance bearings, and prediction of rockslide paths [2-4].

Earlier work on elastoplastic contact deformation focused on the contact of a sphere and a rigid half space to analyze contact between identical spheres [5-8]. In these models, several empirical parameters are used to fit experimental curves and capture aspects of the deformation process that cannot be directly derived analytically. Such models have been used to inform experiments in a limited range of material properties, geometries, or loading cases [9-14], but their restrictive assumptions make them unsuitable for a general predictive analysis. Furthermore, a model that is dynamically valid for materials that exhibit strain rate dependence has been elusive.

In this paper, we propose an elastoplastic contact model, which incorporates material strain rate dependence. The model extracts the quasistatic contact response from finite-element (FEM) analysis by inputting only the material properties and geometry of the spheres. The strain rate dependence is incorporated in the model by introducing a Johnson-Cook type strain-rate dependence into the elastic-perfectly plastic material model determined through FEM analysis. We validate this model with experiments where the contact between two spheres is excited dynamically in a Hopkinson bar. We test the model for a range of materials and loading conditions, varying the spheres material properties and striker bar velocities.

\section{MODEL DESCRIPTION}

The quasistatic compression of an elastoplastic spherical contact has three distinct regimes as shown in Fig. 1(a) [12]. In region I, no part of the sphere has yielded and the forcedisplacement relationship is the elastic Hertzian description.
Region II begins when yield first occurs in the sphere at a relative approach between the centers of the spheres $\delta_{y}$. The plastic region develops initially below the surface and is fully contained within regions that are still elastic [15]. The forcedisplacement response in region II can no longer be captured by the Hertzian description, but is not yet linear as initially assumed by early models [16]. The linear region III begins when the plastic zone reaches the edge of contact surface and becomes unconstrained, at a displacement $\delta_{p}$. The plastic zone in each of the three regions is shown with representative FEM results in Fig. 1(b).

In region I, the behavior is accurately described by the analytical solution found by Hertz [17]. The pressure over the contact area is proportional to $\sqrt{a^{2}-r^{2}}$ where $a$ is the contact radius and $r$ is the polar coordinate. Integrating the pressure field over the contact region shows that the normal force, $F$, is proportional to the distance between the centers of the spheres, $\delta$, to the three-halves power. The force in region $\mathrm{I}$ is given by $F=(4 / 3) E \sqrt{r} \delta^{3 / 2}$, with the effective Young's modulus $E=\left[\left(1-v_{1}^{2}\right) / E_{1}+\left(1-v_{2}^{2}\right) / E_{2}\right]^{-1}$, where $E_{1}, E_{2}, v_{1}, v_{2}$ are the Young's moduli and the Poisson's ratio of the two materials, and the effective radius as $r=\left(1 / r_{1}+1 / r_{2}\right)^{-1}$, where $r_{1}, r_{2}$ are the radii of the two spheres [17]. The Hertzian region ends when plasticity is first observed which is determined using the von Mises criterion to be at a displacement $\delta_{y}$ and force $F_{y}$ given by [5] $\delta_{y}=(1 / 4)\left(r / E^{2}\right)\left(1.6 \pi \sigma_{y}\right)^{2}, F_{y}=$ $(1 / 6)(r / E)^{2}\left(1.6 \pi \sigma_{y}\right)^{3}$. Here, $\sigma_{y}$ is the yield stress of the material and the constant 1.6 is determined by maximizing the $\mathrm{J} 2$ invariant of the axisymmetric stress distribution with respect to both angle and depth below the surface.

The onset of plasticity defines the beginning of region II. The pressure field for this region was proposed by Stronge [15] and can be integrated over the contact area to get a forcedisplacement relation of the form $F=\delta(\alpha+\beta \ln \delta)$. $\alpha$ and $\beta$ are obtained by imposing continuity of the force at the boundaries between regions I and III:

$$
\begin{aligned}
& \alpha=\frac{\delta_{p} F_{y} \ln \delta_{p}-\delta_{y} F_{p} \ln \delta_{y}}{\delta_{y} \delta_{p}\left(\ln \delta_{p}-\ln \delta_{y}\right)}, \\
& \beta=\frac{\delta_{y} F_{p}-\delta_{p} F_{y}}{\delta_{y} \delta_{p}\left(\ln \delta_{p}-\ln \delta_{y}\right)} .
\end{aligned}
$$




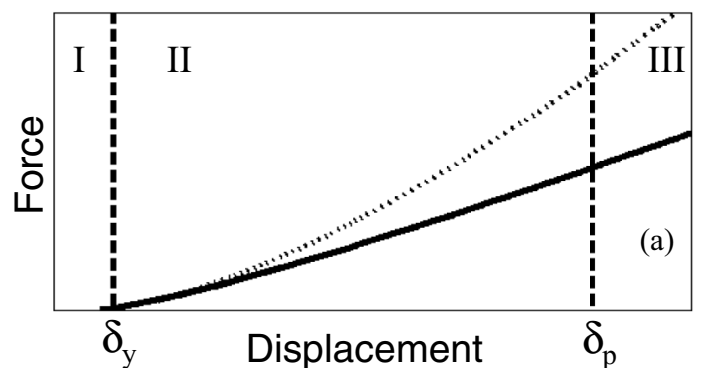

(b)

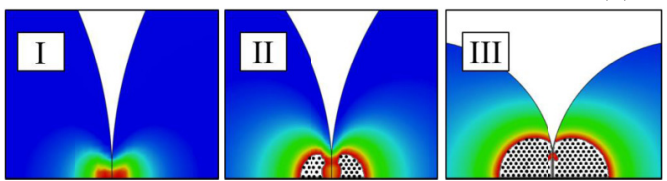

FIG. 1. (Color online) Plot of force versus displacement showing the regions during deformation. Dotted line shows Hertzian solution for elastic spheres. (b) Representative diagrams (obtained via FEM) of the stress distributions in each of the three deformation regions. Dotted region represents the plastic zone.

Since the pressure distribution in region III is nearly constant, the force is equal to the pressure times the area of the contact: $F=p_{0} \pi a^{2}$, where $p_{0}$ is the constant pressure and $a$ is the contact radius $[12,15] . a^{2}$ is modeled by modifying its elastic definition with an empirical parameter, $c_{2}$, that represents the effect of plasticity on the contact radius. The relation is given by Brake as $a^{2}=2 r \delta+c_{2}$ [18]. In previous studies, $p_{0}$ has been assumed to be $2.8 \sigma_{y}$ [18-20]. FEM observations show that this is a reasonable assumption for many cases, but it is not valid for all materials. In this model, we let $p_{0}=c_{1} \sigma_{y}$, where $c_{1}$ is the second empirical parameter.

The displacement at which region III begins, $\delta_{p}$, is the model's third empirical parameter. Given $\delta_{p}$, the force at which region III begins, $F_{p}$, is $F_{p}=p_{0} \pi\left(2 r \delta+c_{2}\right)$.

In order to determine the empirical parameters, material properties are varied within the FEM simulations over a range that includes most metals and common materials [Fig. 2(a)]. The quasistatic model presented here is then fitted to the forcedisplacement results from the FEM simulations. To find $c_{1}$ and $c_{2}$, we choose an arbitrary $\delta_{p}$ and perform a linear regression on the FEM data for $\delta>\delta_{p}$. We calculate the least-squares error in the model versus the FEM results for all possible choices of $\delta_{p}$, and take the empirical constants for which this error is minimized.

The quasistatic model for compressive loading can be summarized in the following piecewise force-displacement relationship:

$$
F(\delta)= \begin{cases}\frac{4}{3} E \sqrt{r} \delta^{3 / 2} & \text { for } \quad 0<\delta<\delta_{y}, \\ \delta(\alpha+\beta \ln \delta) & \text { for } \quad \delta_{y}<\delta<\delta_{p}, \\ p_{0} \pi\left(2 r \delta+c_{2}\right) & \text { for } \delta>\delta_{p} .\end{cases}
$$

Previous works have shown unloading to be a purely elastic process. Here we take the formulation given by Wang [20-23].

With strain-rate-dependent materials, previous FEM analysis has shown that Johnson-Cook strain-rate dependence captures the behavior of moderate-speed sphere impact experiments $[19,24]$. The Johnson-Cook model relates the yield (a)
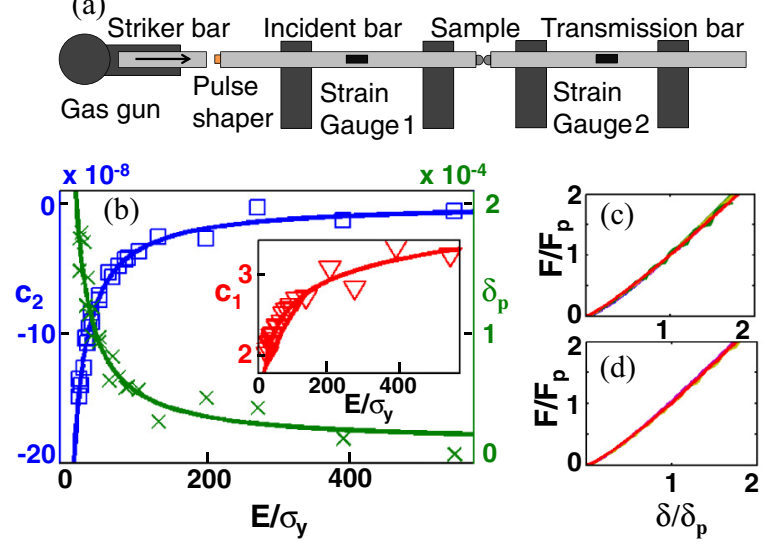

FIG. 2. (Color online) (a) Schematic of split Hopkinson bar experiment. (b) Plot of the model's empirical parameters versus the ratio $E / \sigma_{y}$ with markers showing the results of FEM and the curve fittings used in the model. (c) Multiple plots of normalized force versus displacement taken from FEM results and the results of the model for 4 different values of the ratio $E / \sigma_{y}$. (d) Plots of normalized force versus displacement taken from FEM results and results of the model for sphere diameters $6.35 \mathrm{~mm}, 9.525 \mathrm{~mm}$, and $12.7 \mathrm{~mm}$.

stress to the strain rate as:

$$
\sigma_{y}^{*}=\sigma_{y}\left(1-C \ln \left(\dot{\varepsilon} / \dot{\varepsilon}_{0}\right)\right) .
$$

Here $\dot{\varepsilon}$ is the strain rate, $\dot{\varepsilon}_{0}$ is the quasistatic strain rate at which $\sigma_{y}$ is measured, and $C$ is an empirical parameter determined experimentally [25].

There are two considerations in order to incorporate strainrate dependence into the quasistatic contact model presented above: first, we must define strain rate in the contact problem and, second, we must determine the dependence of the model's parameters on the yield stress of the material. No previous attempt has been made to incorporate rate dependence into an analytical model for the force-displacement relationship, since all knowledge of local strain rates is lost in the analytical description. Here, we define strain rate by considering the relative velocity of approach between the two spheres at each time step as

$$
\dot{\varepsilon}=\frac{v_{2}-v_{1}}{r_{1}+r_{2}},
$$

where $v_{2}$ and $v_{1}$ are the velocities of the centers of mass of the two spheres and $r_{1}$ and $r_{2}$ are the radii of the spheres. We then calculate a new $\sigma_{y}^{*}$ at every time step that is modified by the rate at which the spheres are moving together. Once the dependence on the yield stress is determined, $\sigma_{y}^{*}$ can be used to calculate new dynamic empirical parameters and update the contact model as the simulation progresses.

\section{NUMERICAL AND EXPERIMENTAL SETUP}

To investigate the dependence of the force-displacement response on the material properties and sphere radius, a finite-element model is developed in ABAQUS [26,27]. This allows for the variation of properties over ranges that could not always be explored experimentally. The materials were modeled as elastic-perfectly plastic with Johnson-Cook 
TABLE I. Material properties supplied by manufacturer.

\begin{tabular}{lccc}
\hline \hline & Stainless & Stainless & Aluminum \\
& Steel 440c & Steel 302 & 2017-T4 \\
\hline Density & $7800 \mathrm{~kg} / \mathrm{m}^{3}$ & $7860 \mathrm{~kg} / \mathrm{m}^{3}$ & $2790 \mathrm{~kg} / \mathrm{m}^{3}$ \\
Young's & $204 \mathrm{GPa}$ & $197 \mathrm{GPa}$ & $72 \mathrm{GPa}$ \\
Modulus & $1900 \mathrm{MPa}$ & $600 \mathrm{MPa}$ & $275 \mathrm{MPa}$ \\
Yield Stress & 1900 \\
\hline \hline
\end{tabular}

strain-rate dependence. For quasistatic and constant strain rate simulations we used ABAQUS/Standard, a nonlinear implicit integrator. Truncated spheres [Fig. 1(b)] were placed in hard contact between two rigid analytic surfaces without friction. Final displacements of the rigid surfaces were limited to avoid any building up or sinking in of material around the contact surface. The total duration of the displacement process was varied in order to achieve the desired deformation rate. 1000 displacement steps were used in all simulations. Taking advantage of radial symmetry, $6.35 \mathrm{~mm}$ diameter hemispheres were modeled as quarter circles composed of around 2500 axisymmetric quad elements with nodes at the contact areas spaced $0.025 \mathrm{~mm}$.

Due to the curved geometry and varying contact area inherent in this problem, a fine mesh is required for accurate FEM results. Therefore, FEM quickly becomes very computationally expensive for larger systems beyond two spheres. To extend simulations to the dynamics of multiple spheres, we use the discrete-element method (DEM), implemented here in MATLAB [28-30]. DEM assumes particles to be rigid bodies connected by nonlinear springs governed by a contact interaction model. Writing Newton's second law for each of the particles and contacts determines a system of coupled differential equations that can be solved with conventional numerical methods such as Runge-Kutta integration. DEM sacrifices knowledge regarding local deformations for speed, relying on the contact model to capture the intricacies of the interaction.

To experimentally validate the model at intermediate strain rates, we used a split Hopkinson compression bar [21,31]. A schematic diagram of the experimental setup is presented in Fig. 2(a). Maraging steel bars of $19.05 \mathrm{~mm}$ diameter were used for all bars. Copper pulse shapers were used in order to vary the strain rate and a clay momentum trap was used to prevent reflections. Two identical partial spheres of diameter $6.35 \mathrm{~mm}$ were placed in contact between the incident and transmission bars. In this work, partial spheres of stainless steel 440c, stainless steel 302, and aluminum 2017 were tested with the amplitude of the incident stress wave controlled by varying the pressure driving the striker bar. Using a $10 \mathrm{~cm}$ long striker bar, pressures varied from $50 \mathrm{kPa}$ to $125 \mathrm{kPa}$. Material properties for the various materials are given in Table I.

\section{RESULTS AND DISCUSSION}

Using FEM, we performed quasistatic compression of two $6.35 \mathrm{~mm}$ diameter hemispheres for a variety of material parameters. Each property of each hemisphere was varied independently: Young's modulus, yield stress, and radius. The force-displacement data obtained with FEM were then fitted to the model from Eq. (1) to extract empirical parameters for each simulation [see markers in Fig. 2(b)]. The values of the empirical parameters do not depend on the magnitude of material properties, but rather on the ratio of the effective Young's modulus to the yield stress. After fitting the model to a number of FEM simulations, regression analysis is used to find general empirical functions to describe the parameters' dependence on the ratio of Young's modulus to yield stress [see solid lines in Fig. 2(b)]. The empirical parameters scale with the effective radius as predicted by dimensional analysis, allowing for the FEM results to be scaled to other sphere sizes. Here, these functions were fitted to the compression of $6.35 \mathrm{~mm}$ diameter hemispheres ( $r$ in units of meters):

$$
\begin{aligned}
& \delta_{p}=\left[0.00428\left(\frac{E}{\sigma_{y}}\right)^{-1}+1.47 \times 10^{-5}\right]\left(\frac{r}{0.00159}\right), \\
& c_{1}=-6.76\left(\frac{E}{\sigma_{y}}\right)^{-0.137}+6.304, \\
& c_{2}=\left[-3.99 \times 10^{-6}\left(\frac{E}{\sigma_{y}}\right)^{-1}+1.01 \times 10^{-9}\right]\left(\frac{r}{0.00159}\right)^{2}
\end{aligned}
$$

Using these empirical functions henceforth, we model the quasistatic compression of spheres with different material properties and sizes. Figure 2(c) shows the FEM results and the model for four different values of the ratio of effective Young's modulus to yield stress, normalized by the force and displacement at which region III begins. In each case, the FEM results and the model are nearly indistinguishable. Figure 2(d) shows the normalized FEM and model results for three different sphere radii. In these cases the same empirical functions derived for the $6.35 \mathrm{~mm}$ spheres are used with the appropriate scaling. Again the model results are nearly indistinguishable from the FEM simulations.

With the empirical functions and Eq. (1), we have an explicit relation for the yield stress of the material on the force. Therefore, we can use Eqs. (2) and (3) to modify the model based on velocity of approach of the two spheres. We perform FEM simulations for the compression of two hemispheres with Johnson-Cook strain-rate dependence at a constant relative velocity and compare it with model's predictions. Figure 3(a)

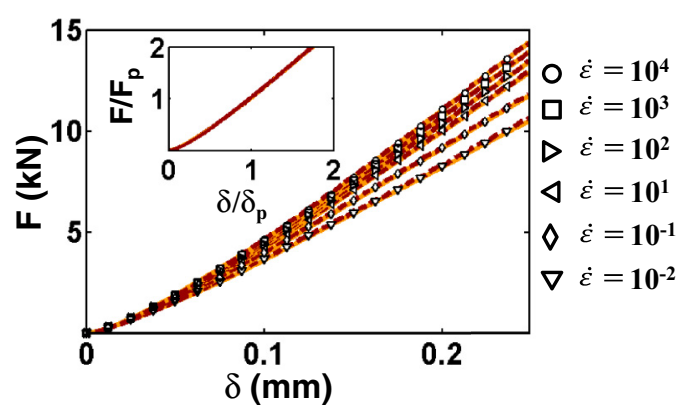

FIG. 3. (Color online) Plot of force versus displacement for compression at various constant strain rates. Dashed dark red (dark gray) lines represent the FEM results and orange (light gray) lines with various markers for each strain rate represent the results from the model. The inset shows the same plots normalized. 

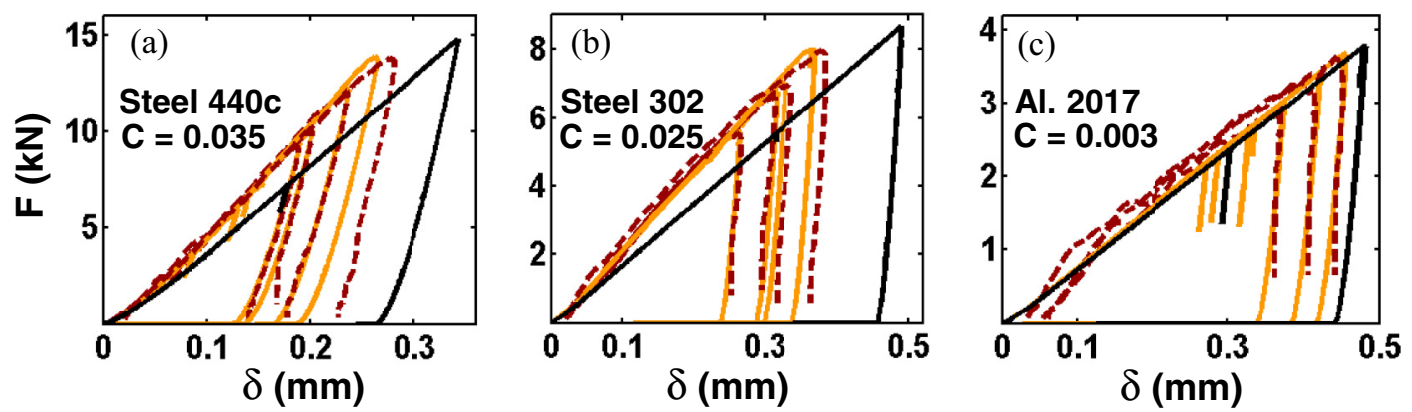

FIG. 4. (Color online) Plots of force versus displacement for experimental results in dashed dark red (dark gray), results using the strain-rate-dependent model for DEM in orange (light gray), and results using the model without strain rate dependence in black. Materials used are (a) stainless steel 440c, (b) stainless steel 302, and (c) aluminum 2017. All results are for 6.35 mm diameter spheres.

shows the results of constant strain-rate FEM analysis versus the analytical model using the same velocity of approach and Johnson-Cook parameters. This figure shows the simulation of $6.35 \mathrm{~mm}$ stainless steel 440c hemispheres with material properties given in Table I and Johnson-Cook parameters: $C=0.04$ and $\dot{\varepsilon}_{0}=0.001$. The inset in Fig. 3(a) shows the same plots normalized by the force and displacement at which region III begins (in this case all curves are overlapping). The model accurately captures the FEM behavior over an extreme range of effective strain rates, from 0.001 (quasistatic) to 10000 [Fig. 3(a)]. Despite losing the information about local deformations and local strain rates, the global strain rate defined above captures the dynamics suitably.

During a Hopkinson bar compression test, strain rate varies greatly. To simulate the event in DEM using the strain-ratedependent empirical model, force versus time measurements taken directly from the experiments were applied to the first particle. The second particle was assumed to be in perfect contact with a one-dimensional linear media with the wave speed and density of the experimental transmission bar.

We compare the DEM simulation's force-displacement results to the Hopkinson bar experimental results for different strain rates and various materials. Figure 4 shows the results of the comparison. The two stainless steels tested (302 and 440c) exhibit relatively large strain-rate dependence while aluminum (2017-T4) shows very little. The Johnson-Cook parameter is chosen to capture the rate dependence and is the same for all simulations of that material. By including dependence on the relative velocity of the two particles, we see that we can effectively simulate the behavior of strain-rate-dependent materials during dynamic events.

\section{CONCLUSIONS}

We have presented a model for the compression of elastoplastic spheres. Once the model's dependence on the yield stress was determined using FEM results, a Johnson-Cook strain-rate dependence was used to modify the effective yield stress based on the relative velocity of the two spheres. This method was shown to agree with both dynamic FEM simulations and experimental results using a Hopkinson bar. Our model captures well the response of the compression of spheres for a number of materials, for different radii spheres, and for a range of loading conditions.

\section{ACKNOWLEDGMENTS}

We would like to thank Prof. Guruswami Ravichandran for discussions and support with experiments, as well as Dr. Dipankar Ghosh and Christian Kettenbeil. This research was supported by Air Force Office of Scientific Research Grant No. FA9550-12-1-0091 through the University Center of Excellence in High-Rate Deformation Physics of Heterogeneous Materials.
[1] H. Hertz, Journal für die reine und angewandte Mathematik 92, 156 (1881).

[2] A. I. Ayesh, S. A. Brown, A. Awasthi, S. C. Hendy, P. Y. Convers, and K. Nichol, Phys. Rev. B 81, 195422 (2010).

[3] J.-M. Hertzsch, H. Scholl, F. Spahn, and I. Katzorke, Astron. Astrophys. 320, 319 (1997).

[4] W. Rammer, M. Brauner, L. K. A. Dorren, F. Berger, and M. J. Lexer, Nat. Hazards Earth Syst. Sci. 10, 699 (2010).

[5] R. L. Jackson and I. Green, J. Tribol. 127, 343 (2005).

[6] L. Kogut and I. Etsion, J. Appl. Mech. 69, 657 (2002).

[7] F. Li, J. Pan, and C. Sinka, J. Mech. Phys. Solids 57, 1194 (2009).

[8] H. Peng, Z. S. Liu, and G. H. Zhang, Proc. Inst. Mech. Eng., Part J 227, 259 (2013).
[9] R. L. Jackson, I. Green, and D. B. Marghitu, Nonlinear Dynamics 60, 217 (2010).

[10] G. Kuwabara and K. Kono, Jpn. J. Appl. Phys. 26, 1230 (1987).

[11] C.-Y. Wu, L.-Y. Li, and C. Thornton, Int. J. Impact Eng. 32, 593 (2005).

[12] K. L. Johnson, Contact Mechanics (Cambridge University Press, Cambridge, 1985), pp. 153-201.

[13] X. Zhang and L. Vu-Quoc, Int. J. Impact Eng. 27, 317 (2002).

[14] R. K. Pal, A. P. Awasthi, and P. H. Guebelle, Granular Matter 15, 747 (2013).

[15] W. J. Stronge, Impact Mechanics (Cambridge University Press, Cambridge, 2000), pp. 116-145.

[16] C. Thornton, J. Appl. Mech. 64, 383 (1997). 
[17] K. Johnson, Contact Mechanics (Cambridge University Press, Cambridge, 1985).

[18] M. R. Brake, Int. J. Solids Struct. 49, 3129 (2012).

[19] H. Minamoto and S. Kawamura, Int. J. Impact Eng. 36, 680 (2009).

[20] C.-y. Wu, L.-y. Li, and C. Thornton, Int. J. Impact Eng. 28, 929 (2003).

[21] E. Wang, P. Geubelle, and J. Lambros, J. Appl. Mech. 80, 021009 (2013).

[22] S. D. Mesarovic and K. L. Johnson, J. Mech. Phys. Solids 48, 2009 (2000).

[23] J. H. Zhao, S. Nagao, and Z. L. Zhang, Int. J. Mech. Sci. 56, 70 (2012).

[24] H. Minamoto and S. Kawamura, Int. J. Impact Eng. 38, 123 (2011).
[25] G. R. Johnson and W. K. Cook, in Proceedings of the Seventh International Symposium on Ballistics, the Hague, the Netherlands, 1983, pp. 541-547, http://www.lajss.org/ HistoricalArticles/A\%20constitutive $\% 20$ model $\% 20$ and\%20data $\% 20$ for\%20metals.pdf.

[26] M. Gonzalez, J. Yang, C. Daraio, and M. Ortiz, Phys. Rev. E 85, 016604 (2012).

[27] S. Hibbitt, ABAQUS/Standard Users Manual, Ver. 5.6, 1996.

[28] V. F. Nesterenko, J. Appl. Mech. Tech. Phys. 24, 733 (1983).

[29] V. F. Nesterenko, Dynamics of Heterogeneous Materials (Springer-Verlag, New York, 2001).

[30] V. F. Nesterenko, C. Daraio, E. B. Herbold, and S. Jin, Phys. Rev. Lett. 95, 158702 (2005).

[31] E. Wang, T. On, and J. Lambros, Exp. Mech. 53, 883 (2012). 